\title{
Immunotherapy in hepatobiliary tumors: search for the missing pieces of the puzzle
}

\author{
Matteo Donadon ${ }^{1,2}$, Federica Marchesi ${ }^{3,4}$, Lorenza Rimassa $^{2,5}$, Guido Torzilli ${ }^{1,2}$ \\ ${ }^{1}$ Department of Hepatobiliary and General Surgery, Humanitas Clinical and Research Center - IRCCS, Rozzano, Milan, Italy; ${ }^{2}$ Department of \\ Biomedical Sciences, Humanitas University, Pieve Emanuele, Milan, Italy; ${ }^{3}$ Department of Immunology and Inflammation, Humanitas Clinical and \\ Research Center - IRCCS, Rozzano, Milan, Italy; ${ }^{4}$ Department of Biotechnology and Translational Medicine, University of Milan, Milan, Italy; \\ ${ }^{5}$ Humanitas Cancer Center, Humanitas Clinical and Research Center - IRCCS, Rozzano, Milan, Italy \\ Correspondence to: Matteo Donadon, MD, PhD. Department of Hepatobiliary and General Surgery, Humanitas University, Humanitas Clinical and \\ Research Center, Via Alessandro Manzoni 56, 20089, Rozzano, Milan, Italy. Email: matteo.donadon@hunimed.eu. \\ Comment on: Sahara K, Farooq SA, Tsilimigras DI, et al. Immunotherapy utilization for hepatobiliary cancer in the United States: disparities among \\ patients with different socioeconomic status. HepatoBiliary Surg Nutr 2020;9:13-24.
}

Submitted Sep 27, 2019. Accepted for publication Oct 24, 2019.

doi: $10.21037 /$ hbsn.2019.10.33

View this article at: http://dx.doi.org/10.21037/hbsn.2019.10.33

In the current era of immunotherapy, the treatment of hepatobiliary cancers is rapidly evolving. The use of immunotherapeutic approaches, which include peptide-based vaccines, checkpoint inhibitors and antibodies, particularly applies to advanced hepatobiliary cancers, for which the availability of limited therapeutic options encourages the adoption of alternative strategies. Thanks to the published/ presented, although conflicting, results of some of the clinical trials on this topic together with the incoming results of some other trials, clinicians involved in the cure of hepatobiliary cancer patients need to understand the basic and advanced applications of immunotherapies (1-6).

On this line, we read with interest the study published by Sahara $e t a l$. (7), who recently reported on the utilization of immunotherapy for hepatobiliary cancers in United States. They identified those patients with a diagnosis of hepatocellular carcinoma (HCC), cholangiocarcinoma or gallbladder cancer treated between 2004 and 2015 that were included in the National Cancer Database, which is a joint venture between the American College of Surgeons and the American Cancer Society. Sahara et al. (7) identified more than 249,000 patients, of which 585 (0.2\%) received immunotherapy. This small percentage is not completely unexpected considering that immunotherapy is not approved for biliary tract cancer patients and has been only recently approved by the United States Food and Drug Administration for previously treated advanced HCC patients, ineligible for potentially curative treatments, such as surgery, liver transplantation and thermal ablation. Based on the collected variables, which included demographics, tumor characteristics, clinical stages, and treatment modalities these authors found some interesting correlations. Expectedly, they found that most of the patients treated by immunotherapy were in advanced stages of their diseases (stage III and IV), which means that they were unsuitable for surgery or loco-regional therapies. Being immunotherapy a systemic therapy, this finding is typical. In addition, they found a favorable trend of utilization of immunotherapy for those patients that were Caucasians, that were treated in academic centers and that had high median income. Yet, some disparities among patients with different socio-economic status were suggested (7). Again, these findings are not totally unexpected given the immunotherapy approval status and the role of academic centers in the development of new therapeutic strategies through clinical trials and easier access to new drugs. Also, socio-economic disparities are not uncommon in the frame of health systems mostly based on private insurances. However, even in countries where national health systems grant a wider access to therapies, disparities related to socio-cultural factors still exist. Indeed, more informed patients are more prone to look for new therapies and to be cured in academic or referral centers.

Many other aspects may influence the availability and the utilization of these novel therapies in hepatobiliary cancer patients. To date, only two anti-PD-1 antibodies, 
namely nivolumab and pembrolizumab, are approved in the United States as second-line therapy for HCC patients, and no checkpoint inhibitors are approved in Europe for the treatment of hepatobiliary cancers. However, although with the above-mentioned differences, some of the reported considerations are valid both in North America and in Europe. The relative scarcity of new agents together with the need to respect the rigorous criteria of the clinical trials may be one explanation for the low rates of utilization of the immunotherapy in hepatobiliary cancers. A second explanation might be found in the clinical fragility that usually is present in hepatobiliary cancer patients. Most of these patients carry an underlying liver disease that limits treatment possibilities, which should be always balanced not to be harmful.

Given the recently presented negative phase 3 trials for HCC (1-6) and waiting for the reading out of the ongoing trials that are testing the combinations of different checkpoint inhibitors or of checkpoint inhibitors with anti-angiogenic drugs, or with chemotherapy or locoregional treatments, further translational research should be carried out aiming to better understand all the relevant implications and to identify potential predictive biomarkers that may help to select which patients may really benefit from these novel therapies $(8,9)$. Indeed, given the scenario of persistent hepatic inflammation that is present in patients with underlying chronic hepatitis or cholestasis there is an increased production of pro-inflammatory and immunosuppressive cytokines and may contribute to the development of a pro-tumor microenvironment, which is unique in comparison with other types of solid tumors (10-12). Yet, very complex interactions among different immune cells, liver cells and cancer cells are present in the liver and they need to be clarified in order to understand first the biology, and second the clinical course of these diseases. Dysregulation of macrophages, CD $8+$ cells, CD4+ cells, CD3+ cells and many other immune types has been reported to various extents, which might be responsible for the wide array of clinical presentations and responsiveness to treatments $(13,14)$. Each of these immune cells provides a nurturing microenvironment for cancers growth and metastasis that represent a key determinant of the efficacy of anticancer strategies. Understanding the interactions between immune and liver cancer cells in general, and in individual patients, should be a priority in cancer research. Such research should be conducted in synergy by hepatobiliary surgeons, medical oncologists, hepatologists, and immunologists to offer an individualized approach, which will likely allow decoding the clinical, pathological, biological and immunological intrigue of hepatobiliary cancers patients. Hopefully, in the near future immunotherapy will be a therapeutic option for a larger number of patients with hepatobiliary cancers and will be offered based on a biological and not socio-economic selection.

\section{Acknowledgments}

None.

\section{Footnote}

Conflicts of Interest: L Rimassa reports receiving consulting fees from Lilly, Bayer, Sirtex Medical, ArQule, Exelixis, Ipsen, Celgene, Eisai, Hengrui Therapeutics, MSD, Baxter, Amgen, Italfarmaco, Sanofi, and Incyte; lecture fees from AstraZeneca, AbbVie, Gilead, and Roche; travel fees from ArQule and Ipsen. The other authors have no conflicts of interest to declare.

Ethical Statement: The authors are accountable for all aspects of the work in ensuring that questions related to the accuracy or integrity of any part of the work are appropriately investigated and resolved.

\section{References}

1. Pardee AD, Butterfield LH. Immunotherapy of hepatocellular carcinoma: Unique challenges and clinical opportunities. Oncoimmunology 2012;1:48-55.

2. El-Khoueiry AB, Sangro B, Yau T, et al. Nivolumab in patients with advanced hepatocellular carcinoma (CheckMate 040): an open-label, non-comparative, phase $1 / 2$ dose escalation and expansion trial. Lancet 2017;389:2492-502.

3. Zhu AX, Finn RS, Edeline J, et al. Pembrolizumab in patients with advanced hepatocellular carcinoma previously treated with sorafenib (KEYNOTE-224): a non-randomised, open-label phase 2 trial. Lancet Oncol 2018;19:940-52.

4. Finn RS, Ryoo BY, Merle P, et al. Results of KEYNOTE-240: phase 3 study of pembrolizumab (Pembro) vs best supportive care (BSC) for second line therapy in advanced hepatocellular carcinoma (HCC). J Clin Oncol 2019;37:Abstract 4004.

5. Available online: https://news.bms.com/press-release/bmy/ 
bristol-myers-squibb-announces-results-checkmate-459study-evaluating-opdivo-nivol

6. Feun LG, Li YY, Wu C, et al. Phase 2 study of pembrolizumab and circulating biomarkers to predict anticancer response in advanced, unresectable hepatocellular carcinoma. Cancer 2019;125:3603-14.

7. Sahara K, Farooq SA, Tsilimigras DI, et al. Immunotherapy utilization for hepatobiliary cancer in the United States: disparities among patients with different socioeconomic status. HepatoBiliary Surg Nutr 2020;9:13-24.

8. Personeni N, Rimassa L. Shaping the landscape of immune oncology in hepatocellular carcinoma. Lancet Oncol 2018;19:855-6.

9. Rimassa L, Personeni N, Aghemo A, et al. The immune milieu of cholangiocarcinoma: From molecular pathogenesis to precision medicine. J Autoimmun 2019;100:17-26.

Cite this article as: Donadon M, Marchesi F, Rimassa L, Torzilli G. Immunotherapy in hepatobiliary tumors: search for the missing pieces of the puzzle. Hepatobiliary Surg Nutr 2020;9(1):86-88. doi: 10.21037/hbsn.2019.10.33
10. Gottlieb A, Best J, Canbay A. Implications of Immunotherapy in Hepatobiliary Tumors. Visc Med 2019;35:18-26.

11. DeLeon TT, Zhou Y, Nagalo BM, et al. Novel immunotherapy strategies for hepatobiliary cancers. Immunotherapy 2018;10:1077-91.

12. Makarova-Rusher OV, Medina-Echeverz J, Duffy AG, et al. The yin and yang of evasion and immune activation in HCC. J Hepatol 2015;62:1420-9.

13. Cortese N, Donadon M, Rigamonti A, et al. Macrophages at the crossroads of anticancer strategies. Front Biosci (Landmark Ed) 2019;24:1271-83.

14. Vigano L, Soldani C, Franceschini B, et al. TumorInfiltrating Lymphocytes and Macrophages in Intrahepatic Cholangiocellular Carcinoma. Impact on Prognosis after Complete Surgery. J Gastrointest Surg 2019. [Epub ahead of print]. 